\title{
General Psychiatry NHS staff mental health status in the active phase of the COVID-19 era: a staff survey in a large London hospital
}

\author{
Ikenna David Ike (D) , Matthieu Durand-Hill, Eiman Elmusharaf, Nicole Asemota, \\ Elizabeth Silva, Elliott White, Wael I Awad
}

To cite: Ike ID, Durand-Hill M, Elmusharaf $\mathrm{E}$, et al. NHS staff mental health status in the active phase of the COVID-19 era: a staff survey in a large London hospital. General Psychiatry 2021;34:e100368. doi:10.1136/ gpsych-2020-100368

- Additional material is published online only. To view, please visit the journal online (http://dx.doi.org/10.1136/ gpsych-2020-100368).

Received 06 August 2020 Revised 30 November 2020 Accepted 18 January 2021

Check for updates

(c) Author(s) (or their employer(s)) 2021. Re-use permitted under CC BY-NC. No commercial re-use. See rights and permissions. Published by BMJ.

Department of Cardiothoracic Surgery, St Bartholomew's Hospital, London, UK

Correspondence to Dr Wael I Awad; wael.awad@nhs.net

\section{ABSTRACT:}

Background Experiencing a pandemic can be very unsettling and may have a negative impact on the mental health of frontline healthcare workers ( $\mathrm{HCWs}$ ). This may have serious consequences for the overall well-being of HCWs, which in turn may adversely affect patient safety and the productivity of the institution.

Aims We designed a study to assess the prevalence of generalised anxiety disorder (GAD), depression and workrelated stress experienced by the National Health Service staff in a large tertiary London hospital treating patients with COVID-19 during the current active phase of the COVID-19 era.

Methods An anonymous survey was designed with demographic data and three questionnaires. The Generalised Anxiety Disorder-7 (GAD-7) and Patient Health Questionnaire-9 were used to assess anxiety and depression, respectively. The Health and Safety Executive Management Standards Indicator Tool was used to assess work-related stress. Staff from multiple specialties embracing cardiothoracic surgery, cardiology, respiratory medicine, endocrinology, oncology, imaging, anaesthesia and intensive care at our hospital were asked to complete the questionnaire between 25 May and 15 June 2020.

Results A total of 302 staff members (106 males and 196 females) completed the survey. The overall prevalence of GAD and depression was $41.4 \%$ and $42.7 \%$, respectively. The prevalence of GAD and depression was significantly higher in females than in males and was statistically significant. Nurses were four times more likely to report moderate to severe levels of anxiety and depression as compared with doctors. Work-related stress was also observed to be prevalent in our surveyed population with the following standards: relationships, role, control and change showing a need for improvement.

Conclusions Our study presents early evidence suggestive of a high prevalence of GAD, depression and work-related stress in HCWs. It is imperative that coherent strategies are implemented to improve the healthcare work environment during this pandemic and mitigate further injury to the mental health status of the healthcare population.

\section{INTRODUCTION}

COVID-19 is a highly infectious and predominantly respiratory illness caused by SARS-CoV-2. To date, there are no available vaccines or specific antiviral treatments for COVID-19, and management involves the treatment of symptoms, supportive care and isolation. WHO declared the COVID-19 outbreak a pandemic on 11 March 2020. ${ }^{1}$ Although WHO reported a mortality rate of $5.22 \%$ for COVID-19, ${ }^{2}$ the UK reported 285 420 confirmed cases with 44220 deaths, on 6 July 2020. ${ }^{3}$ As such, COVID-19 continues to pose a significant and prolonged strain on healthcare systems.

Affected patients tend to be of an older age; of black, Asian and minority ethnic (BAME) ethnicity; in long-term medical facilities such as care homes and with underlying medical conditions, particularly diabetes, hypertension and previous lung and cardiovascular disease. ${ }^{4}$ These comorbidities result in a six times higher risk of hospital admission. ${ }^{5}$ In addition, those affected by COVID-19 are likely to develop venous thromboembolism $(19 \%-85 \%)$, cardiac dysfunction $(7 \%-30 \%)$ and acute kidney injury $(10 \%-30 \%) .^{4-6}$

The COVID-19 pandemic has led to a state of heightened uncertainty in terms of the economy, employment, finances and relationships and has negatively affected the physical and mental health of the general population. ${ }^{7}$ The UK Department of Health and the Confederation of British Industry have estimated that $15 \%-30 \%$ of workers will experience some form of mental health problem during their working lives, ${ }^{8}$ and this is only likely to increase in pandemic environments. Employee performance, rates of illness, absenteeism, accidents and staff turnover are all affected by employees' mental health status. ${ }^{58}$

Anxiety and depression are the most common mental health problems, with prevalence rates of $5.9 \%$ and $3.3 \%$, respectively, in the UK. ${ }^{9}$ They are often a reaction to a difficult life event, such as bereavement or severe illness, but can also be caused or aggravated 
by work-related issues. Previous pandemics have demonstrated the enormous physical, emotional and mental strain placed on healthcare workers (HCWs) during these periods. ${ }^{10}$ This usually manifests in higher rates of anxiety, depression and stress. ${ }^{11}$

Increased workload, physical exhaustion, risk of infection to self (14\% rate of infection among HCWs in the $\mathrm{UK})^{4}$, friends and family, isolation and lack of social support are some of the factors that make HCWs particularly vulnerable. ${ }^{512}$ The impact of COVID-19 on frontline HCWs has been well documented in China, with doctors and nurses showing significantly higher rates of mental health disorders. ${ }^{13}$ Supporting staff's mental well-being is therefore critical to ensuring the sustained health and capacity of the National Health Service (NHS) workforce as they respond to COVID-19.

Furthermore, the UK Health and Safety Executive's (HSE) Management Standards outline six key areas of work that, if poorly managed, ${ }^{14}{ }^{15}$ are associated with diminished work returns, poor health, lower productivity, and increased accident and sickness absence rates. This approach to tackling work-related stress establishes a framework to help employers, including the NHS, tackle work-related stress and, as a result, reduce the incidence and negative impact of mental ill health. Thus, it is imperative that suitable interventions are made to strengthen the psychological well-being and capabilities of healthcare teams, enabling them to continue treating patients to the best of their ability.

However, despite the known mental health repercussions associated with work-related stress, and working in a pandemic, there is a paucity of data on the impact of the current crisis on the mental health of HCWs, with most research on COVID-19 related to clinical consequences of the virus. Moreover, the information that is available has largely been based on Chinese workers, with no assessment of mental health within the western HCW population and specifically in the UK.

The aim of this study was to assess the extent of mental health problems experienced by the NHS staff (both clinical and non-clinical) in the UK during the current active phase of the COVID-19 era. Findings will help determine current working conditions and enable future improvements.

\section{METHODS}

This evaluation was performed between 25 May and 15 June 2020 at a tertiary London hospital with 306 inpatient bed capacity, 55 critical beds, staffed by more than 1100 medical and non-medical HCWs. The research is reported in line with the Standards for Quality Improvement Reporting Excellence guidelines. ${ }^{16}$ A survey was designed with demographic data and three questionnaires, which assessed generalised anxiety disorder (GAD), depression and work-related stress. The questionnaires were distributed all around our hospital, and responses were collected on an anonymous basis (see figure 1).

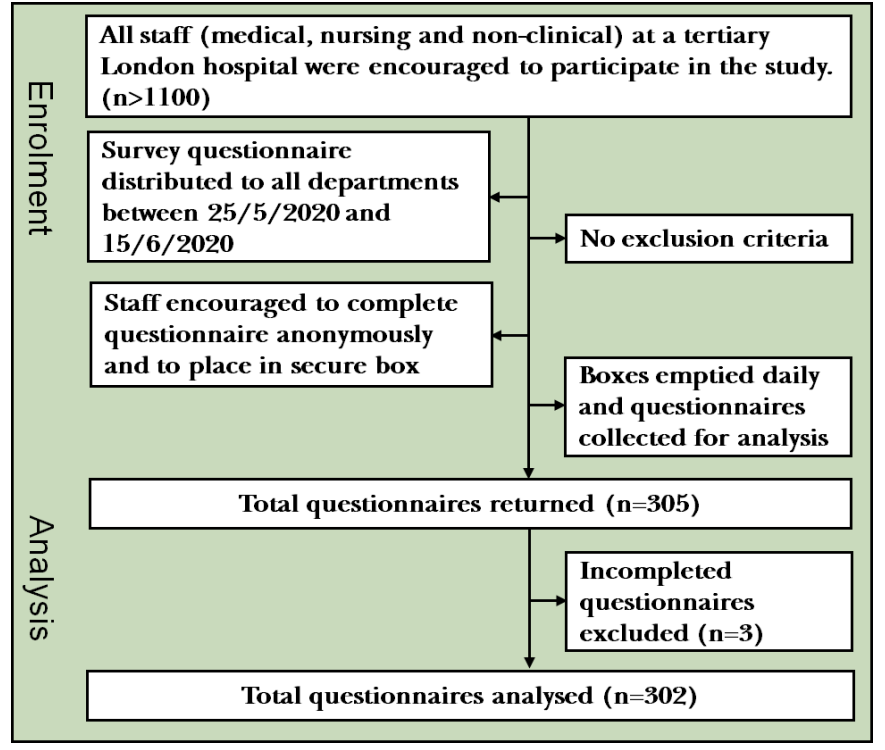

Figure 1 Flowchart for enrolment in the study.

\section{Demographics}

Demographic data collected are shown in online supplemental appendix A. All staff from our hospital were asked to complete the questionnaire and included medical/ nursing staff from multiple specialties embracing cardiothoracic surgery, cardiology, respiratory medicine, endocrinology, oncology, imaging, anaesthesia and intensive care. Allied healthcare professionals including clinical perfusion scientists, operating department personnel, physiotherapists, occupation therapists, pharmacists, lab technicians and other non-medically trained staff were also surveyed. Ethnicity was grouped into (i) BAME and (ii) white, as a number of sources report a much higher risk of COVID-19 infection among BAME staff. ${ }^{17}$ Place of work was divided into four broad categories: intensive therapy unit (ITU), ward, operating theatre and other (imaging department, pharmacy, research and science lab, security and administration).

\section{Generalised Anxiety Disorder-7}

The Generalised Anxiety Disorder-7 (GAD-7) ${ }^{18}$ was used to assess anxiety symptoms (online supplemental appendix B). It has been shown to be reliable and well validated as a measure of anxiety with a Cronbach's score of $0.9 .{ }^{18-20}$ Seven items are assessed by assigning scores of $0,1,2$ and 3 , to the response categories of not at all, several days, more than half the days and nearly every day, respectively. The total score for the seven items ranges from 0 to 21, with scores of 5, 10 and 15 representing cutpoints for mild, moderate and severe anxiety, respectively.

\section{Depression}

We used the Patient Health Questionnaire (PHQ-9) ${ }^{21} 22$ for the assessment of symptoms of depression (online supplemental appendix C). It has been well validated as a diagnostic tool for mental health with Beard et $a l^{23}$ reporting evidence to support its use as a measure of depression severity and treatment outcome. ${ }^{21-23}$ 
Depression severity is calculated by assigning scores of 0 , 1,2 and 3 , to the response categories of not at all, several days, more than half the days and nearly every day, respectively. PHQ-9 total score for the nine items ranges from 0 to 27 . Scores of 5, 10, 15 and 20 represent cut-points for mild, moderate, moderately severe and severe depression, respectively.

\section{Work-related stress}

The HSE's Management Standards Indicator Tool $(\text { HSE-MS IT })^{14}$ has been well validated and its scales show specific sensitivity in the assessment of different aspects of work-related distress, including self-perception of stress at work (online supplemental appendix D). ${ }^{24}$ The management standards are measured by a 35 -item IT, which includes the following domains: demands, control, management support, peer support, relationships, role and change. A 5-point Likert-type scale and a 5-point frequency scale are used. The results are compared with benchmark data provided in the HSE-MS IT manual (online supplemental appendix E). These benchmark data are based on surveys conducted by HSE within 136 organisations and is composed of 'organisational averages'. ${ }^{14}$ These scores are expressed in percentiles and are colour-coded to ease their reading. Scores at or below the 20th percentile indicate the need for urgent action, whereas scores at or above the 80th percentile indicate good performance.

Staff were also given the opportunity to comment on areas of greatest concern to themselves during this era.

\section{Statistical analysis}

Statistical analysis was performed using Prism V.5 (GraphPad). Continuous variables were presented as mean (SD). Categorical variables are shown as the percentage. Categorical variables were evaluated using a $\chi^{2}$ test or Fisher's exact test as appropriate. A p value of $<0.05$ was considered significant for all statistical methods.

\section{RESULTS}

\section{Demographic characteristics}

A total of 302 staff members completed the survey. The characteristics of staff members participating in the survey are shown in table 1. In our study, 96 (31.8\%) participants indicated that they had been tested for COVID-19; $44 \%$ were tested for experiencing COVID-19 symptoms, while $26 \%$ were tested after contact with patients who tested positive for COVID-19. One hundred twenty-seven (42.1\%) participants took time off work during the active phase. The mean length of time off work was 11.8 (8.54) days; $77 \%$ of this subgroup gave COVID-19-related reasons for their absence from work, including being symptomatic, self-isolating or testing positive for the disease.

\section{Anxiety}

The prevalence of symptoms related to GADs, stratified by gender, age, professional role, place of work and
Table 1 Demographic characteristics of NHS staff completing survey $(\mathrm{N}=302)$

\begin{tabular}{lc}
\hline Variable & $\mathbf{n}(\%)$ \\
\hline $\begin{array}{l}\text { Gender } \\
\text { Male }\end{array}$ & $106(35.1)$ \\
\hline Female & $196(64.9)$ \\
\hline Age (years) & \\
\hline $18-29$ & $102(33.8)$ \\
\hline $30-39$ & $94(31.1)$ \\
\hline $40-49$ & $64(21.2)$ \\
\hline $50-59$ & $38(12.6)$ \\
\hline $60+$ & $4(1.3)$ \\
\hline Place of work & \\
\hline Ward & $148(49.0)$ \\
\hline ITU & $66(21.9)$ \\
\hline Theatre & $43(14.2)$ \\
\hline Other & $45(14.9)$ \\
\hline Professional role & \\
\hline Doctors & $62(20.5)$ \\
\hline Nurses & $115(38.1)$ \\
\hline Other & $125(41.4)$ \\
\hline Ethnicity & \\
\hline BAME & $68(55.6)$ \\
\hline White & $134(44.4)$ \\
\hline
\end{tabular}

Contact patients with confirmed COVID-19*

Yes 251 (83.0)

Contact with patients suspected of COVID-19†

Yes $262(86.8)$

${ }^{*}$ Confirmed COVID-19 was defined as patient with COVID-positive viral PCR oropharyngeal swab or radiological features of COVID. †Suspected of COVID-19 was defined as patient with symptoms/ medical history suspicious of COVID but without COVID-positive viral PCR oropharyngeal swab or radiological features of COVID. BAME, black, Asian and minority ethnicity; COVID-19, coronavirus disease 2019; ITU, intensive therapy unit; NHS, National Health Service; PCR, polymerase chain reaction.

ethnicity are shown in table 2. 'Yes' indicates results above threshold for anxiety, and 'no' indicates results below threshold values. The prevalence of symptoms related to GAD within the study population was 125 of $302(41.4 \%$, 95\% CI: $35.8 \%$ to $46.9 \%)$; mild $23.8 \%(72 / 302)$ and moderate/severe $17.6 \%$ (53/302). The prevalence of symptoms related to GAD in consultants $(n=16)$ versus junior doctors $(n=46)$ was $18.8 \%$ vs $34.8 \%$, respectively.

\section{Depression}

The prevalence of symptoms related to depression stratified by gender, age, place of work, professional role and ethnicity are shown in table 3 . 'Yes' indicates results above threshold values for depression, and 'no' indicates results 
Table 2 Data suggestive of generalised anxiety by GAD-7, stratified by gender, professional role, ethnicity, age and place of work

\begin{tabular}{|c|c|c|c|c|c|c|}
\hline \multirow[b]{2}{*}{ Variables } & \multirow[b]{2}{*}{ Total, n (\%) } & \multicolumn{3}{|l|}{ Anxiety } & \multirow[b]{2}{*}{$\chi^{2}$} & \multirow[b]{2}{*}{$P$ value } \\
\hline & & No, n (\%) & Mild, n (\%) & $\begin{array}{l}\text { Moderate to severe, } \\
\mathrm{n}(\%)\end{array}$ & & \\
\hline \multicolumn{7}{|l|}{ Gender } \\
\hline Male & $106(35)$ & 78 (73.6) & $17(16.0)$ & $11(10.4)$ & \multirow[t]{2}{*}{15.2} & \multirow[t]{2}{*}{$<0.001$} \\
\hline Female & $196(65)$ & $99(50.5)$ & $55(28.1)$ & $42(21.4)$ & & \\
\hline \multicolumn{7}{|c|}{ Professional role } \\
\hline Doctors & $62(21)$ & $43(69.4)$ & $15(24.2)$ & $4(6.5)$ & \multirow[t]{2}{*}{9.7} & \multirow[t]{2}{*}{0.008} \\
\hline Nurses & $115(38)$ & $60(52.2)$ & $26(22.6)$ & 29 (25.2) & & \\
\hline \multicolumn{7}{|l|}{ Ethnicity } \\
\hline BAME & $168(56)$ & 98 (58.3) & $41(24.4)$ & 29 (17.3) & \multirow[t]{2}{*}{0.073} & \multirow[t]{2}{*}{0.96} \\
\hline White & $134(44)$ & 79 (59.0) & $31(23.1)$ & $24(17.9)$ & & \\
\hline \multicolumn{7}{|l|}{ Age (years) } \\
\hline $18-29$ & $102(34)$ & $53(52.0)$ & $33(32.4)$ & $16(15.7)$ & \multirow[t]{4}{*}{10.2} & \multirow[t]{4}{*}{0.11} \\
\hline 30-39 & $94(31)$ & $58(61.7)$ & $22(23.4)$ & $14(14.9)$ & & \\
\hline $40-49$ & $64(31)$ & $38(59.4)$ & $13(20.3)$ & $13(20.3)$ & & \\
\hline $50+$ & $42(14)$ & $28(66.7)$ & $4(9.5)$ & $10(23.8)$ & & \\
\hline \multicolumn{7}{|c|}{ Place of work } \\
\hline ITU & $66(22)$ & 37 (56.1) & $17(25.8)$ & $12(18.2)$ & \multirow[t]{4}{*}{1.97} & \multirow[t]{4}{*}{0.92} \\
\hline Theatre & $43(14)$ & $26(60.5)$ & $8(18.6)$ & $9(20.9)$ & & \\
\hline Ward & $148(49)$ & $90(60.8)$ & 35 (23.6) & $23(15.5)$ & & \\
\hline Other & $45(15)$ & 24 (53.3) & $12(26.7)$ & $9(20.0)$ & & \\
\hline
\end{tabular}

The total score for the seven items in the GAD questionnaire ranges from 0 to 21 , with scores of 5,10 and 15 representing cut-points for mild, moderate and severe anxiety, respectively.

BAME, black, Asian and minority ethnicity; GAD-7, Generalised Anxiety Disorder-7; ITU, intensive therapy unit.

below threshold values. The overall prevalence of symptoms related to depression, within the study population was 129 of 302 (42.7\%, 95\% CI: $37.1 \%$ to $48.3 \%)$; mild $25.5 \%$ (77/302) and moderate/severe $17.2 \%$ (52/302). The prevalence of symptoms related to depression in consultants $(n=16)$ versus junior doctors $(n=46)$ was $18.8 \%$ vs $34.8 \%$, respectively.

\section{Work-related stress}

Average working conditions compared with HSE-MS IT are shown in figure 2. Results that fall below the 20th percentile are coloured red and indicate that the average of employees' scores falls below the bottom $20 \%$ of those surveyed in the benchmark data and indicate an urgent need for improvement. Results that are below the 50th percentile but are above the 20th percentile are coloured amber and indicate a need for improvement. Scores that are above the 50th percentile but below the 80 th percentile are colour-coded aqua and indicate good performance with potential for improvement. Results above the 80th percentile are coloured green, which indicates that the scores are in the top $20 \%$ of those surveyed in the benchmark data.

In this survey, employee scores for control and role were below average but above or at the 20th percentile, indicating a need for improvement in these subgroups. Employee scores for each domain, stratified by place of work, are presented in figure 3 . Nurses were observed to score lower than doctors in the domains for demands, peer support and relationships. Both doctors and nurses reported scores that were below the 50th percentile for 'control' and 'change'. However, all scores were between the 20th and 80th percentile of the benchmark data, indicating average performance and a need for improvement in all domains. Females were also observed to score lower than males for work-related stress across all management standards, with 'control' and 'change' being the worst affected standards with scores below the 50th percentile.

\section{Thematic analysis of common complaints and disturbing events}

One hundred seventy-three $(57.2 \%)$ participants in the survey made comments on areas of genuine concern. Table 4 shows the most common concerns expressed by participants in the survey. HCWs expressed their anxiety at the potential risk to families and friends and struggled with having to isolate from loved ones. A further analysis of the comments made by nurses showed a frustration with poor communication from management about new protocols, policies and rota changes. 
Table 3 Data suggestive of depression by PHQ-9, stratified by gender, professional role, ethnicity, age and place of work

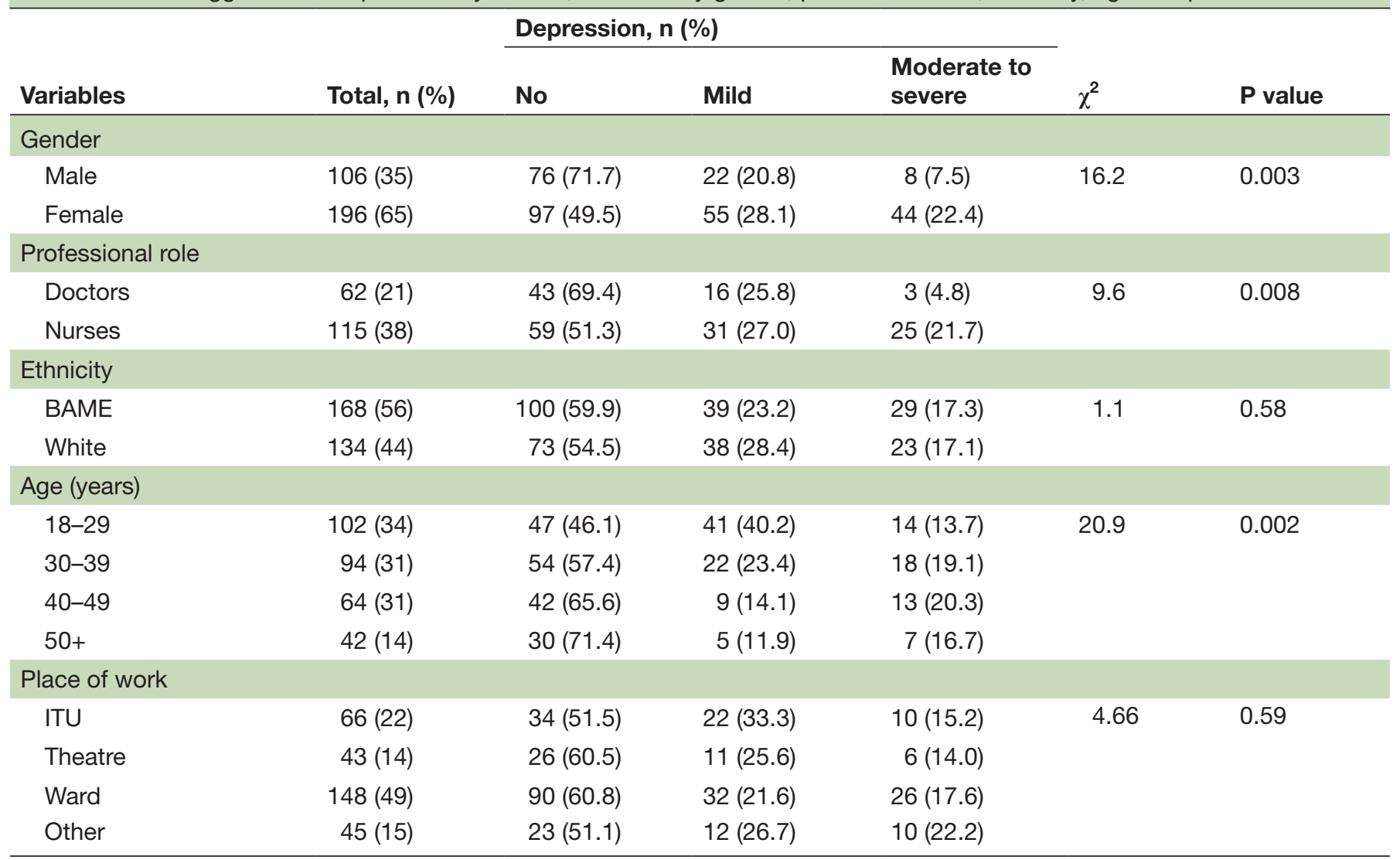

PHQ-9 total score for the nine items ranges from 0 to 27 . Scores of 5, 10, 15 and 20 represent cut-points for mild, moderate, moderately severe and severe depression, respectively.

BAME, black, Asian and minority ethnicity; ITU, intensive therapy unit; PHQ-9, Patient Health Questionnaire.

\section{DISCUSSION}

\section{Main findings}

Our study shows a high prevalence of common mental health disorders in HCWs in our hospital. The overall prevalence of symptoms related to general anxiety and depression indicated by these data was $41.4 \%$ and $42.7 \%$, respectively, which is significantly higher than the rate of $15.7 \%$ reported from the Adult Psychiatry Morbidity Survey $(2014)^{7}$ of the general UK population using the GAD-7 questionnaire. Bentall et al showed an increase in anxiety, depression and stress in the UK during the active phase of COVID-19, reporting anxiety rates of $17.9 \%$ and $25.2 \%$ and depression rates of $20.58 \%$ and $23.4 \%$ in males and females, respectively. ${ }^{7}$

Work-related stress, measured using the HSE-MS IT as shown in figures 1 and 2, was also observed to be prevalent in our surveyed population. This is an important finding as the HSE-MS IT is positively associated with job satisfaction and negatively associated with job-related anxiety and depression. ${ }^{25}$ The standards that showed a need for improvement in our hospital were relationships, role, control and change, with the latter two just at or above the 20th percentile of benchmark scores.

These results suggest that the pandemic may be having a significant impact on the mental health of HCWs in our hospital. There are many factors that may explain these results. Concern for one's own safety and concerns of 'bringing the virus home' to family are often cited as causes of mental anguish. Many authors have shown that the shortage of personal protective equipment (PPE) was a risk factor for detrimental mental health among HCWs. ${ }^{26}{ }^{27}$ A lack of PPE was often cited as a common complaint in our survey, most especially by nurses.

Furthermore, redeployment and lack of certainty regarding job roles was often quoted as a cause of mental anguish, with other studies also demonstrating the detriment of redeployment on mental health. ${ }^{28}$ This was further exacerbated by a lack of adequate support and training before transfer to unfamiliar environments. Redeployment also exacerbated concerns of lack of control and high demands, which were recurring themes expressed by staff.

Further evaluation of the survey data revealed a significant difference in the rates of common mental disorders when stratified by gender. Females were twice more likely than males to experience symptoms of anxiety and depression. This demonstrates an already established gender difference in the prevalence of mental health disorders. ${ }^{129}$

Questionnaire data for nurses also suggested significantly higher rates of GAD and depression than doctors. They were four times more likely to report moderate to 


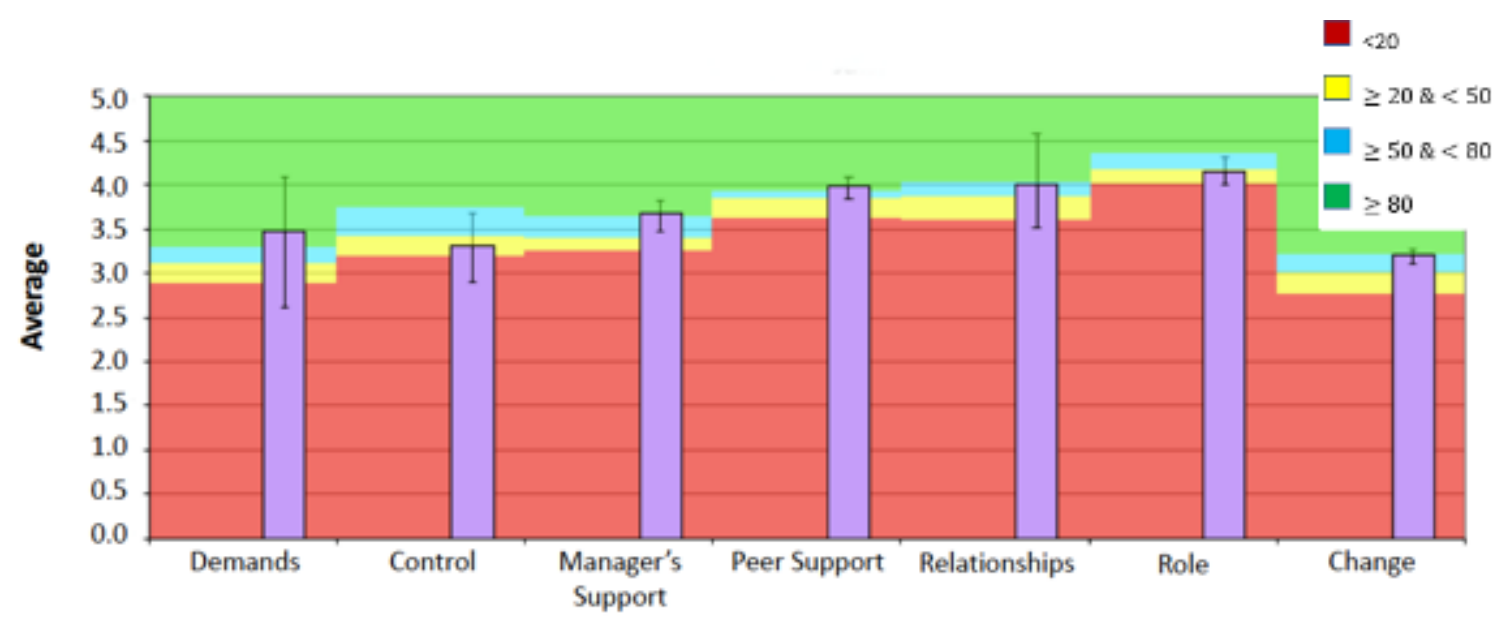

Management Standard

T Range of question averages

$\perp$ for specified factor

\section{Percentile key}

\begin{tabular}{|l|l|}
\hline Below $20^{\text {th }}$ percentile (bottom $20 \%$ of benchmark scores)/Urgent action required \\
\hline & $\begin{array}{l}\text { Below average but above or at } 20^{\text {th }} \text { percentile/Improvement needed } \\
\text { improvement }\end{array}$ \\
\hline $\begin{array}{l}\text { Above or at } 80^{\text {th }} \text { percentile (top } 20 \% \text { of benchmark)/Doing very well - Need to } \\
\text { maintain performance }\end{array}$ \\
\hline
\end{tabular}

Figure 2 Summary of results for management standards as surveyed by the HSE-MS IT. HSE-MS IT, Health and Safety Executive Management Standards Indicator Tool.

severe levels of anxiety and depression as compared with doctors. This may be confounded by the higher proportion of nurses being female, ${ }^{12}$ but can also be explained by their propensity to be in closer contact with patients and for longer periods than doctors.

Nurses reported a frustration with poor communication from management about new protocols, policies and rota changes. This perceived lack of leadership may explain the results seen in the HSE-ME IT, where nurses reported poorer satisfaction with workplace demands, control and support provided.

Reduced rates of GAD and depression among doctors in our hospital may be explained by better support and a more robust leadership structure for doctors than nurses. Nevertheless, there was a difference between junior doctors and consultants, with junior doctors experiencing a higher prevalence of anxiety and depression.

We also observed a significant difference in the prevalence of scores suggesting depression with respect to age, with younger HCWs showing higher rates of depression and a linear decrease in depression rates with increasing age, as shown in table 3 . However, there was no difference in the rates of generalised anxiety with age. These results may be explained by stronger resilience in the older population, as they have acquired more experience over the course of their careers and may have experienced previous pandemics in the past.

There was no relationship between possible anxiety and depression with place of work and ethnicity, suggesting a widespread and relatively similar impact of COVID-19 on all departments and staff of different backgrounds. It is also important to note that ethnicity has little influence on the prevalence of anxiety, depression and work stress, suggested by questionnaire data, despite reports of a higher risk of coronavirus infection among BAME staff (four times higher than when compared with non-BAME counterparts). ${ }^{17}$

Although place of work was not an independent predictor of anxiety and depression, theatre staff appeared to report higher levels of distress in all HSE domains, compared with staff from other places of work. This may be attributed to the central role that our hospital played during this COVID-19 crisis, whereby the unit was one of only two units providing emergency cardiothoracic surgery to the whole of the London population, as other units reduced their workload to cope with increasing 


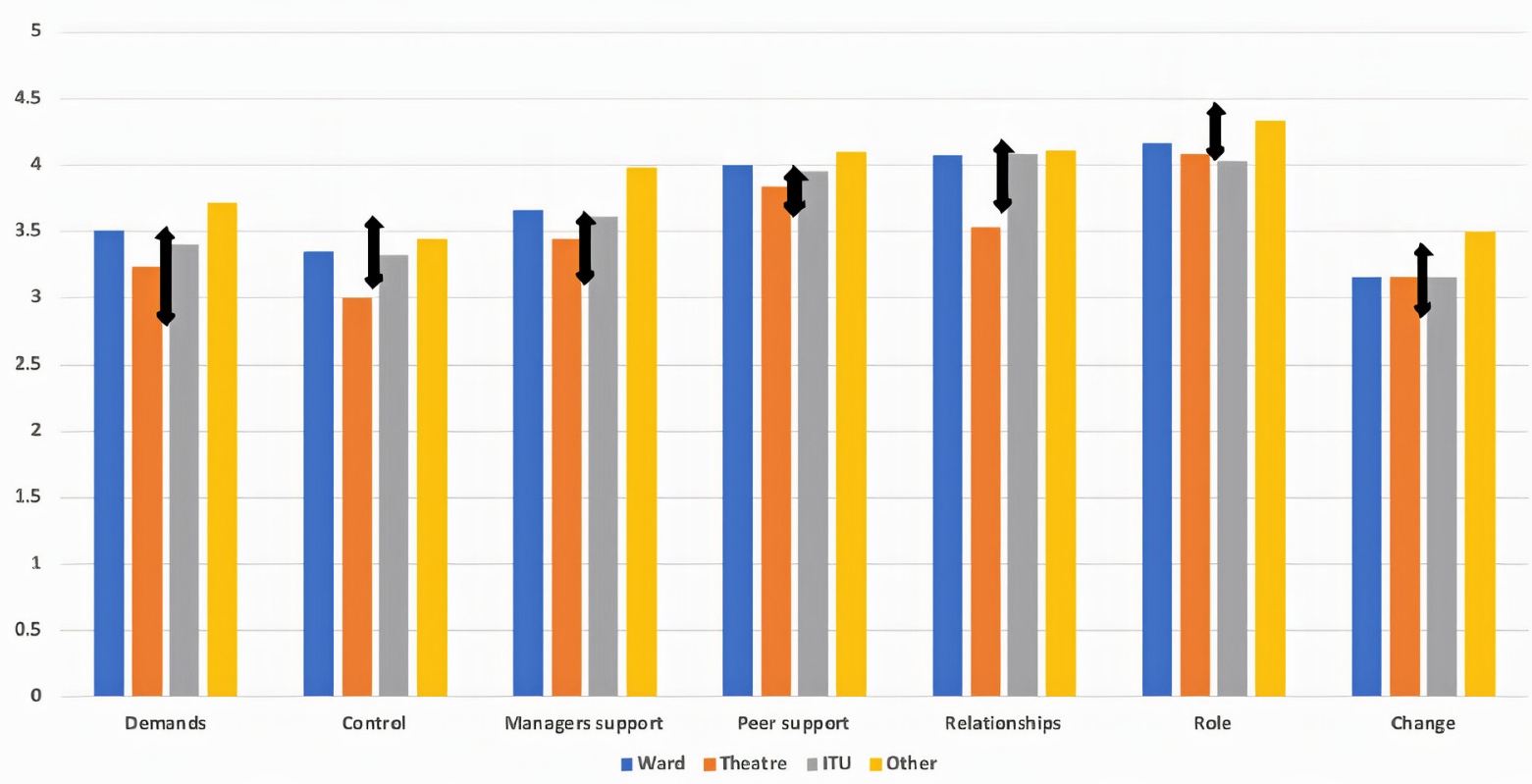

Figure 3 Health and safety executive average scores stratified by place of work. HSE-MS IT, Health and Safety Executive Management Standards Indicator Tool; ITU, intensive therapy unit. I Range of averages for each management standard subgroup as compared with benchmark data; 'organisational averages' of 136 institutions surveyed by HSE-MS IT. ${ }^{14}$ Top arrow denotes $80^{\text {th }}$ percentile and above, while bottom arrow denotes $20^{\text {th }}$ percentile and below.

patients with COVID-19. Dramatic changes to standard working practices, alongside other stressors described will potentially cause these concerns.

Working in a pandemic has been shown to present new and particular ethical dilemmas among the NHS staff, including the so-called 'moral injury'. 1228 These moral dilemmas include the shortage of staff in critical care areas, inability to have physical contact with patients, rationing of medical supplies including ventilators and, in one's personal life, isolation from family for protection. These ethical and moral dilemmas are likely to exacerbate ongoing mental anguish, predisposing HCWs to depression and anxiety, and are much harder to resolve. Nurses

Table 4 Thematic analysis of frequent comments made by surveyed participants ( $\mathrm{N}=173$ )

\begin{tabular}{|c|c|}
\hline Themes & n (\%) \\
\hline Inadequate PPE for staff & $42(24.2)$ \\
\hline $\begin{array}{l}\text { Lack of leadership, guidance and support } \\
\text { from senior management }\end{array}$ & $33(19.0)$ \\
\hline $\begin{array}{l}\text { Redeployment to ITU without appropriate } \\
\text { training and support }\end{array}$ & $28(16.1)$ \\
\hline Concern for patients' well-being & $28(16.1)$ \\
\hline Concern about family and friends & $22(12.7)$ \\
\hline $\begin{array}{l}\text { Unclear changes in hospital policies and } \\
\text { guidelines }\end{array}$ & 19 (10.9) \\
\hline Lack of testing for staff & $15(8.6)$ \\
\hline Concern for personal well-being & $12(6.9)$ \\
\hline Impact of high mortality and morbidity & $5(2.8)$ \\
\hline
\end{tabular}

ITU, intensive therapy unit; PPE, personal protective equipment. are also likely more vulnerable to moral injury ${ }^{12}{ }^{28}$, as they witness the consequences and sequelae of COVID-19 disease in affected patients.

Given the unique nature of this crisis and its potential to persist for an extended time period, it is imperative that suitable interventions are made to strengthen the psychological resilience and capacity of healthcare systems. It is pertinent that those in managerial responsibility instigate steps to mitigate these effects. These include, but are not limited to, increased support for critical care staff, areas of rest for staff members and provision of emotional support for workers.

Senior support is paramount to effective working, particularly in these unknown and uncomfortable environments, with clear channels of communication (regular departmental meetings, huddles, emails and newsletters) aimed to maintain good morale among all workers. Teams could also be made more resilient by these measures and by providing a safe environment to speak out, reduced hierarchies and inequalities, devolved and autonomous decision making where roles lead to a sense of belonging and competence. Although many measures to improve mental health may be provided, it is still the responsibility of the individual to use the services offered. As such, it is important to provide a work environment that encourages the individual to seek help when their mental health is at risk. ${ }^{28}$

Frameworks to aid with the creation of robust support systems have been developed. The British Psychological Society outlined three phases of psychological impact on the NHS staff as they respond to COVID-19, which employers should consider when developing and implementing robust and comprehensive mental well-being support mechanisms. ${ }^{30}$ 
1. Preparation phase: early in the course of the pandemic with staff waiting in 'anticipation'. Limited input and time for planning can result in staff feeling unprepared.

2. Active phase. sustained pressure on staff lasting weeks and possibly months. Staff will swing from a feeling of 'heroics' and 'surge to action' towards disillusionment and exhaustion. This is the highest period of psychological risk where staff may neglect their physical and psychological self by putting their work above their own well-being.

3. Recovery phase: staff will experience recovery and, in some cases, potentially the long-term psychological impacts of the outbreak. Having time to reflect, some individuals may experience a sense of regret over what they 'should' have done differently and shame or guilt. Some may be at risk of chronic mental health concerns including post-traumatic stress disorder. We must therefore recognise that there will be no respite for HCWs as the pandemic moves into the recovery phase.

\section{Limitations}

This study does not separate those with pre-existing mental health issues, in particular, by ascertaining a baseline for participants. Furthermore, this study is a single-centre analysis of a London hospital and may not be a true reflection of the greater NHS staff population. Finally, an element of selection bias may be inadvertently introduced, as it is possible that those with the greatest concerns were either more likely to complete the questionnaires, or, conversely, least likely to admit to these issues. Similarly, it is possible that those whose mental health concerns were not significant may therefore have been less likely to complete the questionnaire. Low responses and non-responses to our survey may have led to an underestimation of negative responses, potentially introduced bias, impacting the representativeness of the sample and skewing results.

\section{Implications}

This study provides a much-needed insight into the work environment in a busy NHS hospital during COVID-19, and our detailed analysis of the prevalence of anxiety, depression and work-stress stratified by gender, age, professional roles, place of work and ethnicity gives valuable information that may be representative of the experiences of the NHS in general. Our data suggest a high prevalence of generalised anxiety, depression and workrelated stress in HCWs. It also elucidates key factors and areas that will be of great importance for health policy makers and governing institutions as they seek coherent strategies and bespoke interventions to maintain and improve the HCW environment during this pandemic and mitigate further injury to the mental health status of the healthcare population.

Contributors WIA introduced the concept. IDI, MD-H, EE and WIA designed the study. IDI, MD-H, EE and WIA did the literature search. IDI, MD-H, EE, NA and WIA supervised the study and data collection. IDI and MD-H analysed and interpreted the data. IDI, MD-H and NA wrote the first draft. ES, EW and WIA suggested improvements. IDI, MD-H, EE and NA wrote the final draft. IDI, MD-H and EE made the tables. IDI and WIA reviewed the manuscript. WIA supervised the publication.

Funding The authors have not declared a specific grant for this research from any funding agency in the public, commercial or not-for-profit sectors.

Competing interests None declared.

Patient consent for publication Not required.

Ethics approval The study was registered with the Integrated Research Application System (IRAS project ID: 282914) and reviewed by the Health Research Authority (HRA) and local Research Ethics Committee and did not require ethical approval, as participation was voluntary and the completed surveys were anonymised. The study was also approved by the Barts NHS Trust Quality Improvement Committee (ID 11259).

Provenance and peer review Not commissioned; externally peer reviewed.

Data availability statement Data are available on reasonable request. Please contact corresponding author if data are required.

Supplemental material This content has been supplied by the author(s). It has not been vetted by BMJ Publishing Group Limited (BMJ) and may not have been peer-reviewed. Any opinions or recommendations discussed are solely those of the author(s) and are not endorsed by BMJ. BMJ disclaims all liability and responsibility arising from any reliance placed on the content. Where the content includes any translated material, BMJ does not warrant the accuracy and reliability of the translations (including but not limited to local regulations, clinical guidelines, terminology, drug names and drug dosages), and is not responsible for any error and/or omissions arising from translation and adaptation or otherwise.

Open access This is an open access article distributed in accordance with the Creative Commons Attribution Non Commercial (CC BY-NC 4.0) license, which permits others to distribute, remix, adapt, build upon this work non-commercially, and license their derivative works on different terms, provided the original work is properly cited, appropriate credit is given, any changes made indicated, and the use is non-commercial. See: http://creativecommons.org/licenses/by-nc/4.0/.

ORCID iD

Ikenna David Ike http://orcid.org/0000-0003-0893-5233

\section{REFERENCES}

1 World Health Organisation. WHO Director-General's opening remarks at the media briefing on COVID-19 - 11 March 2020. Available: https://www.who.int/dg/speeches/detail/who-director-general-sopening-remarks-at-the-media-briefing-on-covid-19-11-march-2020

2 World Health Organisation. WHO coronavirus disease (COVID-19) Dashboard, 2020. Available: https://covid19. who.int/?gclid=CjOK CQjwl4v4BRDaARIsAFjATPmYmvpo9N-0cml4Cxwrj8Xg6hYrweE mPCcnzSP7wspMPYgvatk2ZzEaAt1rEALw_wcB

3 World Health Organisation. The United Kingdom: WHO coronavirus disease (COVID-19) Dashboard. Available: https://covid19.who.int/ region/euro/country/gb

4 Beeching NJ, Fletcher TE, Fowler R. Coronavirus disease 2019 (COVID-19) - Symptoms, diagnosis and treatment. BMJ Best Practice 2020 https://bestpractice.bmj.com/topics/en-gb/3000168

5 Phua J, Weng L, Ling L, et al. Intensive care management of coronavirus disease 2019 (COVID-19): challenges and recommendations. Lancet Respir Med 2020;8:506-17.

6 Petrosillo N, Viceconte G, Ergonul O, et al. COVID-19, SARS and MERS: are they closely related? Clin Microbiol Infect 2020;26:729-34.

7 Shevlin M, Nolan E, Owczarek M, et al. COVID-19-related anxiety predicts somatic symptoms in the UK population. Br J Health Psychol 2020;25:875-82.

8 Liimatainen M-R, Gabriel P. Mental health in the workplace: situation analyses. United Kingdom Int Labour Off; 2020. https://www.ilo.org/ wcmsp5/groups/public/-ed_emp/-ifp_skills/documents/publication/ wcms_108226.pdf

9 Edwards J, Goldie I, Elliott I. Fundamental facts about mental health Mental Health Foundation; 2016.

10 Liu X, Kakade M, Fuller CJ, et al. Depression after exposure to stressful events: lessons learned from the severe acute respiratory syndrome epidemic. Compr Psychiatry 2012;53:15-23.

11 Wu P, Fang Y, Guan Z, et al. The psychological impact of the SARS epidemic on hospital employees in China: exposure, risk perception, and altruistic acceptance of risk. Can J Psychiatry 2009;54:302-11. 
12 Pappa S, Ntella V, Giannakas T, et al. Prevalence of depression, anxiety, and insomnia among healthcare workers during the COVID-19 pandemic: a systematic review and meta-analysis. Brain Behav Immun 2020;88:901-7.

13 Huang C, Wang Y, Li X, et al. Clinical features of patients infected with 2019 novel coronavirus in Wuhan, China. The Lancet 2020;395:497-506. doi:10.1016/S0140-6736(20)30183-5

14 HSE. What are the management standards? 2020. Available: https:// www.hse.gov.uk/stress/standards/

15 Cousins R, MacKay CJ, Clarke SD, et al. 'Management Standards' work-related stress in the UK: practical development. Work Stress 2004;18:113-36. doi:10.1080/02678370410001734322

16 Ogrinc G, Davies L, Goodman D, et al. SQUIRE 2.0 (Standards for QUality Improvement Reporting Excellence) : revised publication guidelines from a detailed consensus process. BMJ Qual Saf 2016;25:986-92. doi:10.1136/bmjqs-2015-004411

17 Public Health England. Disparities in the risk and outcomes from COVID-19, 2020. Available: www.facebook.com/PublicHealthEngland

18 Spitzer RL, Kroenke K, Williams JBW, et al. A brief measure for assessing generalized anxiety disorder: the GAD-7. Arch Intern Med 2006;166:1092-7.

19 Löwe B, Decker O, Müller S, et al. Validation and standardization of the generalized anxiety disorder screener (GAD-7) in the general population. Med Care 2008;46:266-74.

20 Tong X, An D, McGonigal A, et al. Validation of the generalized anxiety Disorder-7 (GAD-7) among Chinese people with epilepsy. Epilepsy Res 2016;120:31-6.
21 Kroenke K, Spitzer RL, Williams JB. The PHQ-9: validity of a brief depression severity measure. J Gen Intern Med 2001;16:606-13.

22 Kroenke K, Spitzer RL. The PHQ-9: a new depression diagnostic and severity measure. Psychiatr Ann 2002;32:509-15.

23 Beard C, Hsu KJ, Rifkin LS, et al. Validation of the PHQ-9 in a psychiatric sample. J Affect Disord 2016;193:267-73.

24 Marcatto F, Colautti L, Larese Filon F, et al. The HSE management standards indicator tool: concurrent and construct validity. Occup Med 2014;64:365-71.

25 Kerr R, McHugh M, McCrory M. HSE management standards and stress-related work outcomes. Occup Med 2009;59:574-9.

26 Chung JPY, Yeung WS. Staff mental health self-assessment during the COVID-19 outbreak. East Asian Arch Psychiatry 2020;30:34.

27 Lu W, Wang H, Lin Y, et al. Psychological status of medical workforce during the COVID-19 pandemic: a cross-sectional study. Psychiatry Res 2020;288:112936.

28 Greenberg N, Docherty M, Gnanapragasam S, et al. Managing mental health challenges faced by healthcare workers during covid-19 pandemic. BMJ 2020;368:m1211.

29 Albert PR. Why is depression more prevalent in women? J Psychiatry Neurosci 2015;40:219-21.

30 British Psychological Society COVID 19 Staff Wellbeing Group. The psychological needs of healthcare staff as a result of the coronavirus pandemic, 2020. Available: www.ics.ac.uk/ICS/Education/Wellbeing/ ICS/Wellbeing.aspx

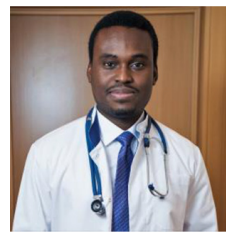

Dr Ikenna David obtained his MBBS (Hons) from the Pirogov Russian National Research Medical University, Russia, in 2016. He is currently a cardiothoracic clinical fellow at St Bartholomew's Hospital, Barts NHS Trust, London, the UK. He founded the Barts Association of Cardiothoracic Researchers for Junior Doctors and is the current president of the Association. He is also a co-founder of the London School of Cardiothoracics, a non-profit organisation that provides education in cardiothoracic surgery to the UK medical practitioners. He has participated and presented in various national and international conferences. His main research interests include aortic surgery research, surgical innovation and perioperative psychiatry. 